American Journal of Biochemistry and Biotechnology 6 (2): 120-135, 2010

ISSN 1553-3468

(C) 2010 Science Publications

\title{
The Development of a Revised Canadian Myalgic Encephalomyelitis Chronic Fatigue Syndrome Case Definition
}

\author{
${ }^{1}$ Leonard A. Jason, ${ }^{1}$ Meredyth Evans, ${ }^{1}$ Nicole Porter, ${ }^{1}$ Molly Brown, \\ ${ }^{1}$ Abigail Brown, ${ }^{1}$ Jessica Hunnell, ${ }^{1}$ Valerie Anderson, ${ }^{1}$ Athena Lerch, \\ ${ }^{2}$ Kenny De Meirleir and ${ }^{3}$ Fred Friedberg \\ ${ }^{1}$ Department of Psychology, DePaul University, \\ Center for Community Research, Chicago, Il, 60614, USA \\ ${ }^{2}$ Department of Physical Education and Physical Therapy, \\ Vrije Universiteit Brussel, Belgium \\ ${ }^{3}$ Department of Psychiatry and Behavioral Science, \\ State University of New York at Stony Brook, USA
}

\begin{abstract}
Problem statement: Several investigators have indicated that case definitions for Chronic Fatigue Syndrome (CFS) are characterized by vaguely worded criteria that lack operational definitions and guidelines. The most widely used CFS case definition is the Fukuda et al. criteria, which uses polythetic criteria (i.e., patients are only required to have four out of a possible eight symptoms). Yet two of these eight symptoms (post-exertional malaise and memory/concentration problems) are an essential feature of this illness and the Fukuda et al. criteria do not require that these symptoms be present among all patients. Significant methodological problems could occur if investigators in different settings recruit samples with different percentages of these core symptoms. In contrast, the Canadian clinical case definition does require specific ME/CFS symptoms such as post-exertional malaise and memory/concentration problems. The provision of operationally explicit, objective criteria on specific key symptoms might reduce criterion variance as a source of unreliability. In addition, the use of structured interview schedules will ensure that symptoms are assessed in a consistent way across settings. Conclusion/Recommendations: In this article, we specified explicit rules for determining whether critical symptoms meet ME/CFS criteria using a revised Canadian case definition and a questionnaire has been developed to assess core symptoms. It is hoped that these developments will lead to increased reliability of this revised Canadian case definition as well as more frequent use of these criteria by investigators.
\end{abstract}

Key words: Myalgic encephalomyelitis, chronic fatigue syndrome, chronic fatigue, post-exertional malaise, illness

\section{INTRODUCTION}

Efforts to develop a CFS case definition can be traced back to the 1950s. In 1955, there was an outbreak of a CFS-like illness at the Royal Free Hospital and Ramsay (1981; 1988), the medical consultant in charge, published a number of descriptions of this disease, which subsequently became known as Myalgic Encephalomyelitis (ME) (Hyde et al., 1992) (Ramsay used a different term in his early research, but he changed after Acheson (1959) came up with ME). Based on Ramsay's concept, research criteria were developed by Dowsett et al. (1990) and (1994) and recently revised by Goudsmit et al. (2009) in an effort to distinguish the ME criteria from that of CFS. These ME case definitions recognize the following four cardinal features: (1) physical or mental fatigue or muscle weakness after minimal exertion which may persist long after exertion ends; (2) circulatory impairment (e.g., feeling hot when it's cold, postural hypotension); (3) one or more symptoms indicating the involvement of the central nervous system such as impairment of memory and concentration and disturbed sleep patterns and (4) the marked fluctuation of symptoms. Other symptoms emphasized included: Pain and autonomic and

Corresponding Author: Leonard A. Jason, Department of Psychology, DePaul University, Center for Community Research, Chicago, Il, 60614, USA Tel: 773-325-2018 Fax: 773-325-4923 
immunological abnormalities and physicians were alerted to inappropriate night or daytime sweating, gastro-intestinal disturbances, dizziness or vertigo, hyperacusis, blurred vision, sore throat, headaches and intolerance to alcohol. When Jason et al. (2003) attempted to operationalize some of these ME criteria by selecting individuals with postexertional malaise, memory and concentration impairment and fluctuation of symptoms and then compared these patients to those meeting the current US definition of CFS (Fukuda et al., 1994), the ME criteria selected a more symptomatic group of patients.

In 1998, Holmes et al. (1988) constructed the first US working case definition of CFS. According to this case definition, individuals needed to report six or more months of persistent or relapsing, debilitating fatigue that does not resolve with bedrest to meet criteria. Also, participants were required to report at least 8 of 11 minor symptoms (fever or chills, sore throat, lymph node pain, muscle weakness, muscle pain, post-exertional malaise, headaches of a new or different type, migratory arthralgia, neuropsychiatric complaints, sleep disturbance and a sudden onset of symptoms). Participants were also required to report at least a 50\% impairment of daily functioning, as compared to premorbid levels. As the Holmes et al. (1988) criteria were utilized in research and practice, it became evident that there were numerous inconsistencies in interpretation and classification. For example, Katon et al. (1991) found that patients with CFS were indistinguishable from those with chronic fatigue not meeting the (Holmes et al., 1988) CDC criteria. Another major concern was that the requirement of eight or more minor symptoms could inadvertently select for individuals with psychiatric problems (Katon and Russo, 1992).

A few years later, another set of more broadly defined CFS criteria were developed by British researchers (Sharpe et al., 1991). To qualify for a diagnosis of CFS using the British CFS criteria, the following features needed to be present: (1) fatigue must be the principal symptom; (2) the syndrome must be definite in onset and not lifelong; (3) the syndrome must be severe, disabling and have an effect on physical and mental (cognitive) functioning; (4) the syndrome must have been present for six months or more than $50 \%$ of the time and (5) other symptoms may be present, particularly myalgias, mood and sleep disturbance. These criteria were not frequently used by investigators, as they were considered considerably broader than the Holmes et al. (1988) criteria and a few years later, international consensus developed a new CFS case definition (Fukuda et al., 1994).
In 1994, with sponsorship from the Centers for Disease Control and prevention (CDC) in the US, a revised case definition for CFS (Fukuda et al., 1994) was developed and it is now used throughout the world. This CFS case definition requires a person to experience six or more months of chronic fatigue of new or definite onset, that is not substantially alleviated by rest, not the result of ongoing exertion and that results in substantial reductions in occupational, social and personal activities. To be diagnosed with CFS, individuals also need to have the concurrent occurrence of four or more of eight symptoms that do not predate the fatigue and persists for six or more months since the onset (e.g., sore throat, lymph node pain, muscle pain, joint pain, post-exertional malaise, headaches of a new or different type, memory and concentration difficulties and unrefreshing sleep). Although the first CFS criteria published by Holmes et al. (1988) (as specified by the Schluederberg et al. (1992) revision), excluded individuals with the presence of anxiety disorders, somatoform disorders and nonpsychotic or nonmelancholic depression prior to CFS onset, these conditions were no longer exclusionary under the Fukuda et al. case definition. Jason et al. (2001) compared the Fukuda et al. (1994) and Holmes et al. (1988) criteria and found that the Holmes et al. (1988) criteria selected a group of patients with higher symptomatology and functional impairment.

Unfortunately, the current US case definition for CFS (Fukuda et al., 1994) is characterized by vaguely worded criteria that are lacking operational definitions and guidelines to assist health care professionals in their interpretation and application of the diagnostic tool (Jason et al., 1999a; Reeves et al., 2003). In order to provide more guidelines and specific criteria for this case definition, the CDC developed an empiric case definition for CFS that involves assessment of symptoms, disability and fatigue (Reeves et al., 2005). The CDC empiric case definition assesses disability using the Medical Outcomes Study Short Form-36 Health Survey (SF-36) (Ware et al., 2000); symptoms using the Symptom Inventory (Wagner et al., 2005) and fatigue using the Multidimensional Fatigue Inventory (Smets et al., 1995). However, using these new empiric criteria, the estimated prevalence rates of CFS have increased to $2.54 \%$ (Reeves et al., 2007), rates that are about ten times higher than prior CDC estimates (Reyes et al., 2003) and prevalence estimates of other investigators (Jason et al., 1999b) using the Fukuda et al. criteria. It is possible that the increase in CFS prevalence in the United States is due to a broadening of the Fukuda et al. case definition in an attempt to operationalize the criteria. Further, the 
empiric case definition has potential for inclusion of cases with primary psychiatric conditions. In support of this thesis, Jason et al. (2009a) found that 38\% of those with a diagnosis of a Major Depressive Disorder were misclassified as having CFS using the new CDC empiric case definition.

The Fukuda et al. (1994) case definition uses polythetic criteria, that is, a set of symptoms in which not all need to be present to make a diagnosis. The use of polythetic criteria derived by expert committees (as is the case with CFS) may not be methodologically sound (Jason and Choi, 2008). For example, use of polythetic criteria may result in the comparison of two different groups of patients within the same diagnostic category or similar groups in different diagnostic categories. Because the Fukuda et al. (1994) criteria only require four symptoms out of a possible eight, critical CFS symptoms such as post-exertional malaise and memory and concentration problems are not required of all patients and this might further complicate identification of comparable samples. In contrast, a CFS case definition developed in Australia by Lloyd et al. (1990) stipulated that post-exertional malaise, as well as memory and concentration difficulties were central for a diagnosis (this definition has not been frequently used, as is true with the British criteria). As mentioned above, the earlier ME definition involves two primary symptoms: post-exertional malaise and impairment of memory and concentration (Dowsett et al., 1994).

A clinical case definition for ME/CFS that is called the Canadian criteria also specified the these two core symptoms as well as several other symptoms (Carruthers et al., 2003). (The acronym ME/CFS refers to Myalgic Encephalomyelitis and Chronic Fatigue Syndrome, according to the Canadian Case Definition. The patient community has felt that the term chronic fatigue syndrome trivializes the seriousness of this illness, as the illness is typified by many severe symptoms in addition to fatigue and fatigue is generally regarded as a common symptom experienced by many otherwise healthy individuals in the general population. The term Myalgic Encephalomyelitis had been used prior to the use of the term chronic fatigue syndrome (Acheson, 1959). The Canadian clinical case definition specifies that post-exertional malaise must occur with a loss of physical or mental stamina, rapid muscle or cognitive fatigability, usually taking 24 hours or longer to recover. In addition, there need to be two or more neurological/cognitive manifestations (e.g., confusion, impairment of concentration and short term-memory). There also needs to be unrefreshing sleep or poor sleep quantity or rhythm disturbance, as well as a significant degree of arthralgia and/or myalgia (there are a small number of patients with no pain or sleep dysfunction and a diagnosis can only be given when these individuals have a classical case with an infectious illness onset). Finally, there needs to be at least one symptom from two of the following categories: autonomic manifestations (neurally mediated hypotension, light headedness), neuroendocrine manifestations (e.g., recurrent feelings of feverishness and cold extremities) and immune manifestations (e.g., recurrent sore throats). Jason et al. (2004) compared persons meeting the Canadian clinical case definition (Carruthers et al., 2003), the Fukuda et al. criteria and people experiencing chronic fatigue explained by psychiatric reasons. The Canadian criteria, in contrast to the Fukuda et al. criteria, selected cases with less psychiatric comorbidity, more physical functional impairment, more fatigue/weakness and neuropsychiatric and neurology symptoms. Moreover, those meeting the Canadian criteria were more symptomatically different from the psychiatricallycaused chronic fatigue group than those meeting the Fukuda et al. (1994) criteria.

Jason et al. (2006) later used the Canadian case definition model to develop a pediatric case definition for ME/CFS. To meet criteria, youth needed to meet the following six classic categories including fatigue, postexertional malaise; unrefreshing sleep, or disturbance of sleep quantity or rhythm; myofascial pain, joint pain, abdominal and/or head pain; two or more neurological/cognitive manifestations and at least one symptom from two of three subcategories including autonomic manifestations, neuroendocrine manifestations, or immune manifestations. In order to operationalize these symptoms, a questionnaire was developed with specific questions relating to each of the categories above. Jason et al. (2010a) found that the Fukuda et al. (1994) criteria was less sensitive than the Pediatric ME/CFS criteria in identifying pediatric ME/CFS cases. Jason et al. (2010a) developed a separate classification for those who met almost all criteria termed the Moderate ME/CFS clinical criteria. The group that meets full criteria might be most appropriately used for research criteria, whereas those with more moderate features are less impaired and more heterogeneous and thus, they might fall under a more clinical case definition.

More restrictive or more liberal criteria clearly have an effect on who is classified as having CFS but these different definitions also pose difficulties in interpreting results of related studies (Komaroff et al., 1996). To deal with these problems, there have been efforts to use statistical methods to classify symptoms 
of individuals with severe fatigue (Janal et al., 2006; Nisenbaum et al., 1998; 2004; Sullivan et al., 2005). For example, Friedberg et al. (2000) found three symptom factors (cognitive problems, flu-like symptoms and neurological symptoms) in a sample of patients with CFS. Also using factor analysis with patients diagnosed with CFS, Ray et al. (1992) found the following factors: Emotional distress, fatigue, somatic symptoms and cognitive difficulty. Rowe and Rowe (2002) confirmatory factor analysis with a pediatric sample found muscle pain and fatigue, neurological/cognitive symptoms, abdominal, head and chest pain and both neurophysiological and immunological factors.

Some of the theoretical benefits of using these types of statistical approaches to better understand symptoms can be demonstrated from a study by Jason et al. (2002a). They used factor analysis to provide support for the existence of four distinct components of chronic fatigue: Lack of Energy (fatigue intensity), Physical Exertion (fatigue exacerbated by physical exertion), Cognitive Problems (difficulties with short-term memory, concentration and information processing) and Fatigue and Rest (rest or sleep is not restorative). Two of the primary dimensions of fatigue that emerged in a sample meeting the Fukuda et al. (1994) criteria for CFS based on self-report ("CFS-like") were post-exertional fatigue and cognitive problems. Using cluster analysis, Jason and Taylor (2002) found that a majority of individuals with CFS with moderate to severe symptoms could be accurately classified into two clusters both with severe post-exertional fatigue but different levels of improvement after rest. This study also found more severe cognitive problems in the clusters of patients having CFS. These finding support the designation of post-exertional fatigue and cognitive problems as core symptoms, similar to what has been recommended in the ME criteria (Dowsett et al., 1994), the Australian case definition (Lloyd et al., 1990) and the Canadian clinical case definition for ME/CFS (Carruthers et al., 2003).

Finally, Jason et al. (2007) provided individuals with CFS a theoretically driven questionnaire that featured neuropsychiatric, vascular, inflammatory, muscle/joint, infectious and other symptoms. When these theoretically derived symptoms were factor analyzed, a more interpretable factor structure was identified than when factor analysis was applied to the eight Fukuda et al. (1994) symptoms. The findings from this study provide empirical support for identifying the following six types of symptoms among those with CFS: neurocognitive, vascular, inflammation, muscle/joint pain, infectious and sleep/post-exertional malaise. Several studies have provided empirical support for examining these different types of symptoms among patients with CFS including post-exertional malaise (Jason et al., 2002a; Jason and Taylor, 2002), neuropsychological or cognitive problems (Ray et al., 1992; Rowe and Rowe, 2002; Jason et al., 2002a), infectious symptoms (Nisenbaum et al., 1998; Friedberg et al., 2000; Rowe and Rowe, 2002), muscle/joint difficulties (Nisenbaum et al., 2004; Rowe and Rowe, 2002), vascular issues (Rowe and Rowe, 2002; Jason et al., 2002b), inflammatory problems (Corradi et al., 2006) and unrefreshing sleep (Nisenbaum et al., 2004; Jason et al., 2002a). It is interesting that the Canadian clinical case definition (Carruthers et al., 2003) requires all six areas to be assessed, whereas vascular and inflammatory processes are not assessed by the Fukuda et al. CFS criteria. These findings suggest that a broader group of symptoms, ones which are theoretically derived, might be needed to more accurately identify critical symptoms of those with this illness.

The Canadian case definition requires specific ME/CFS symptoms. However, the provision of operationally explicit, objective criteria is not enough to ensure that scientists can elicit the necessary information to permit them to gather reliable information. There is also a need to construct structured interview schedules that ensure that questions are asked in a consistent way and this increases the chance that data collected in different settings are comparable. This report specifies explicit rules for determining ME/CFS status using a revised Canadian case definition and a questionnaire to assess symptoms (for a copy, write the first author).

The revised Canadian ME/CFS criteria: The definition presented in Table 1 has elements of the Fukuda et al. (1994) case definition, along with some of the recommendations of Reeves et al. (2003). We have also incorporated the structure of the Canadian clinical case definition for ME/CFS developed by Carruthers et al. (2003). We believe that requiring specific symptoms does capture the critical symptoms for a person with ME/CFS. However, we have tried to limit the types of symptoms within each of the Canadian criteria categories to allow investigators to more reliably categorize adult patients. 
Table 1: Criteria for the revised Canadian ME/CFS criteria

\section{Definition of Research ME/CFS criteria}

I. Over the past 6 months, persistent or recurring chronic fatigue that is not lifelong and results in substantial reductions in previous levels of occupational, educational, social and personal activities. The concurrent occurrence of the following classic ME/CFS symptoms (See II through VI), which must have persisted or recurred during the past six months of illness (symptoms may predate the reported onset of fatigue).

II. Post-exertional malaise and/ or post-exertional fatigue. With activity (it need not be strenuous and may include walking up a flight of stairs, using a computer, or reading a book), there must be a loss of physical or mental stamina, rapid/sudden muscle or cognitive fatigability, postexertional malaise and/or fatigue and a tendency for other associated symptoms within the patient's cluster of symptoms to worsen. The recovery is slow, often taking 2-24 hours or longer.

III. Unrefreshing sleep or disturbance of sleep quantity or rhythm disturbance. May include unrefreshing sleep, prolonged sleep (including frequent naps), disturbed sleep (e.g., inability to fall asleep or early awakening) and/or day/night reversal.

IV. Pain (or discomfort) that is often widespread and migratory in nature. At least one symptom from any of the following:

Myofascial and/or joint pain. Myofascial pain can include deep pain, abdomen/stomach pain, or achy and sore muscles. Pain, stiffness, or tenderness may occur in any joint but must be present in more than one joint and lacking edema or other signs of inflammation.

Abdominal and/or head pain. May experience stomach pain or chest pain. Headaches often described as localized behind the eyes or in the back of the head. May include headaches localized elsewhere, including migraines. Headaches would need to be more frequent than they were before, which would indicate new pattern, of a new type as compared to headaches previously experienced (i.e., location of pain has changed, nature of pain has changed), or different in severity type as compared to headaches previously experienced by the patient.

V. Two or more neurological/cognitive manifestations:

- Impaired memory (self-reported or observable disturbance in ability to recall information or events on a short-term basis)

- Difficulty focusing vision and attention (disturbed concentration may impair ability to remain on task, to screen out extraneous/excessive stimuli)

Loss of depth perception

- $\quad$ Difficulty finding the right word

- Frequently forget what wanted to say

- $\quad$ Absent mindedness

- Slowness of thought

- $\quad$ Difficulty recalling information

- Need to focus on one thing at a time

- Trouble expressing thought

- Difficulty comprehending information

- Frequently lose train of thought

- Sensitivity to bright lights or noise

- Muscle weakness/muscle twitches

VI. At least one symptom from two of the following three categories:

1. Autonomic manifestations: Neurally mediated hypotension, postural orthostatic tachycardia, delayed postural hypotension, palpitations with or without cardiac arrhythmias, dizziness or fainting, feeling unsteady on the feet--disturbed balance, shortness of breath, nausea, bladder dysfunction, or irritable bowel syndrome.

2. Neuroendocrine manifestations Recurrent feelings of feverishness and cold extremities, subnormal body temperature and marked diurnal fluctuations, sweating episodes, intolerance of extremes of heat and cold, marked weight change-loss of appetite or abnormal appetite.

3. Immune manifestations: Recurrent flu-like symptoms, non-exudative sore or scratchy throat, repeated fevers and sweats, lymph nodes tender to palpitation--generally minimal swelling noted, new sensitivities to food, odors, or chemicals.

VII. Exclusionary versus Non-Exclusionary conditions:

A. Exclusionary conditions:

1. Any active medical condition that may explain the presence of chronic fatigue, such as:

i. $\quad$ Untreated hypothyroidism

ii. Sleep apnea

iii. Narcolepsy

iv. Malignancies

v. Leukemia

vi. Unresolved hepatitis

vii. Multiple Sclerosis

viii. Juvenile rheumatoid arthritis

ix. Lupus erythematosus

x. HIV/AIDS

xi. Severe obesity (BMI greater than 40; but if weight gain follows onset of ME/CFS, the patient could meet the Clinical Criteria)

xii. Celiac disease

xiii. Lyme disease 
Table 1: Continued

2. Some active psychiatric conditions that may explain the presence of chronic fatigue, such as:

i. Schizophrenia or psychotic disorders

ii. Bipolar disorder

iii. Active alcohol or substance abuse-except as below:

a. Alcohol or substance abuse that has been successfully treated and resolved should not be considered exclusionary.

iv. Active anorexia nervosa or bulimia nervosa-except as below:

b. Eating disorders that have been treated and resolved should not be considered exclusionary.

v. Depressive disorders with melancholic or psychotic features

B. Not necessarily exclusionary

3. May have presence of concomitant disorders that do not adequately explain fatigue and are, therefore, not necessarily exclusionary.

i. Psychiatric diagnoses such as:

a. Anxiety disorders

b. Somatoform disorders

c. Depressive disorders

ii. Other conditions defined primarily by symptoms that cannot be confirmed by diagnostic laboratory tests, such as:

a. Multiple food and/or chemical sensitivity

b. Fibromyalgia

iii. Any condition under specific treatment sufficient to alleviate all symptoms related to that condition and for which the adequacy of treatment has been documented.

iv. Any condition that was treated with definitive therapy before development of chronic symptomatic sequelae.

v. Any isolated and unexplained physical examination, laboratory or imaging test abnormality that is insufficient to strongly suggest the existence of an exclusionary condition.

The Canadian ME/CFS clinical case definition (Carruthers et al., 2003), states that "The patient must have a significant degree of new onset, unexplained, persistent, or recurrent physical and mental fatigue that substantially reduces activity level" (p.6) and "the fatigue should be severe enough to substantially reduce the patient's activity level, usually by approximately $50 \%$ ( illness usually has a distinct onset (although it may be gradual) and it persists for at least 6 months.

The Fukuda et al. (1994) case definition states the following: A case of the chronic fatigue syndrome is defined by the presence of the following: (1) clinically evaluated, unexplained, persistent or relapsing chronic fatigue that is of new or definite onset (has not been lifelong); is not the result of ongoing exertion; is not substantially alleviated by rest and results in substantial reduction in previous levels of occupational, educational, social, or personal activities and (2) the concurrent occurrence of four or more of the following symptoms, all of which must have persisted or recurred during 6 or more consecutive months of illness and must not have predated the fatigue (p.956).

Given that some language used in the Fukuda et al. criteria is vague, we have created operational definitions to improve diagnostic reliability.

According to the Fukuda et al. (1994) criteria, a 6 month period could have been over the last 6 months, or during a 6 month period a few years ago. We believe that the survey item should specify that these symptoms should be assessed over the last 6 months in order to determine a current ME/CFS diagnosis. It is also unclear what is meant by "persisted or recurred." This phrase has generally referred to a symptom that has been ongoing and constant, but sometimes there are good periods and bad periods. We now operationalize this phrase by assessing how often the person has experienced the symptom (fatigue or other symptoms) over the past 6 months using the following scale: $0=$ none of the time, $1=$ a little of the time, $2=$ about half the time, $3=$ most of the time, $4=$ all of the time. To be counted as "persisted or recurred," the individual would have to indicate a score of 2 or higher. In addition, it is important that fatigue and the other core symptoms should be either moderate or severe; however this "severity index" has not been well defined in previous criteria. We now specify that existing symptoms be rated on the following scale: $0=$ symptom not present, $1=$ mild, $2=$ moderate, $3=$ severe, $4=$ very severe. Symptoms need to be rated at moderate (i.e., 2) or worse to meet criteria. Therefore, to meet criteria for a given symptom, specific frequency and severity ratings must be met over the past 6 months.

The threshold number of 4 out of 8 Fukuda et al. (1994) core symptoms has been changed and we now adopt a similar system to that used with the Canadian ME/CFS criteria (Carruthers et al., 2003). The DePaul Symptom Questionnaire provides a structured way to gather standardized information that can be used to aid 
in the diagnosis of ME/CFS. For meeting full criteria of $\mathrm{ME} / \mathrm{CFS}$, the following six classic ME/CFS symptom categories must occur. A scoring sheet for determining if a person meets ME/CFS criteria and how to score the SF-36 scales can also be obtained by writing the first author.

Fatigue: Our first criterion involves persistent or recurring chronic fatigue over the past six months that is not lifelong and results in substantial reductions in previous levels of occupational, educational, social and personal activities. The Fukuda et al. (1994) case definition requires that the fatigue not be lifelong and that it be of a new and definite onset. In a revision of the Fukuda et al. (1994) criteria, Reeves et al. (2003) stated that only participants who recount having always felt severely fatigued should be excluded as having "lifelong" fatigue. We also decided to use this criterion in formulating the diagnostic criteria. A person would not meet this ME/CFS criterion if they answered yes to the following question: "Have you always had persistent or recurring fatigue/energy problems, even back to your earliest memories as a child? By persisting or recurring, we mean that the fatigue/energy problems are usually ongoing and constant, but sometimes there are good periods and bad periods. The original Canadian criteria mentioned a "distinct onset" but allowed for gradual onset. We agree with this guideline, but also note that onset often occurs quickly (within days/weeks), especially following an infectious epsiode/surgery/pregnancy. This is not always when patients get all the symptoms but they notice a distinct difference in their health.

In terms of fatigue severity ratings, a number of fatigue scales have been used, but Stouten (2005) has warned that many fatigue scales do not accurately represent the severe fatigue that is characteristics of ME/CFS. Deciding on which subscales and cut off scores to use has also been problematic. For example, with the CDC empiric case definition, Reeves et al. (2005) used the Multidimensional Fatigue Inventory (MFI) (Smets et al., 1995) to assess severe fatigue. The MFI scales range from 4-20 and Reeves et al. (2005) defined severe fatigue as a score of greater than or equal to 13 on the MFI general fatigue scale or greater than or equal to 10 on the reduced activity scale. In one study of three groups with CFS, the mean MFI general fatigue scores were 18.3-18.8 (Tiersky et al., 2003) and these scores are clearly higher than the Reeves et al. (2005) cutoff of 13 . In addition, reduced activity items refer to issues that a person with depression might easily endorse. If a person indicated that the following two items were entirely true: "I get little done," and "I think I do very little in a day," they would meet criterion for fatigue on this scale. Clearly, almost all individuals with depressive disorders would meet this reduced activity criterion. Jason et al. (2010b) also found that Reeves et al. (2005) cutoff scores for the MFI had inadequate sensitivity and specificity when attempting to differentiate ME/CFS cases from controls.

In order to avoid the potential pitfalls associated with previous approaches of operationalizing fatigue and to maintain consistency with the Fukuda et al. (1994) and Carruthers et al. (2003) case definitions, the $\mathrm{ME} / \mathrm{CFS}$ fatigue criterion is met if a person indicates that their fatigue/extreme tiredness has persisted or recurred over the past 6 months at frequency and severity ratings of 2 or higher. A person must also meet the criteria for substantial reductions described below. Some patients with CFS are not chronically fatigued, but they have a problem of endurance or stamina and need lengthy times to recover following minimal degrees of activity (Hyde, 1999). A person with ME/CFS who participates in very little activity (possibly to minimize ME/CFS symptoms) when compared to his or her same-age peers, could become exhausted upon minimal exertion. While normal fatigue is not activity limiting, the fatigue present in ME/CFS limits the individual's activity to varying degrees. Therefore, in addition to assessing whether or not a person has persistent or recurring chronic fatigue over the past 6 months, we have added questions that identify those individuals who have low stamina and endurance, but currently have less fatigue/energy problems because they are severely limiting their daily activities. Individuals who do not meet the fatigue criterion may still obtain a Clinical ME/CFS diagnosis if they meet the other five criteria.

Substantial reduction in functioning: According to the Fukuda et al. (1994) criteria, fatigue is associated with substantial reductions in "previous levels of occupational, educational, social, or personal activities" (p.956). Therefore, we have included an assessment of substantial reductions under the fatigue criterion. An instrument that has been frequently used to assess this disability construct is the Medical Outcomes Study Short Form-36 Health Survey (SF-36) (Ware and Sherbourne, 1992). This instrument is a 36 item, broadlybased, self report measure of functional status related to physical and social functioning, physical role functioning, emotional role functioning, bodily pain, general health, vitality, mental health and health transition. Higher scores on this scale indicate higher functioning.

Reeves et al. (2005) empiric CFS case definition selected four of the eight SF-36 subscales to measure 
disability/substantial reductions. According to Reeves et al. (2005) empiric case definition, the disability criterion was met by scoring below the 25 th percentile on at least one of the following four SF-36 sub-scales: Physical Functioning (less than or equal to 70), Role-Physical (less than or equal to 50), Social Functioning (less than or equal to 75), or RoleEmotional (less than or equal to 66.7). Based on this system, a person could meet the disability CFS criteria without any reductions in key areas of physical functioning and only have impairment in the role emotional area (e.g., problems with work or other daily activities as a result of emotional problems). Jason et al. (2009a) have challenged the inclusion of the RoleEmotional subscale as not being appropriate because it assesses change in function as a result of any emotional problems. Ware et al. (2000) found that the mean for Role-Emotional for a clinical depression group was 38.9, indicating that almost all those with clinical depression would meet the CFS disability criterion, as they would be within the lower 25 th percentile on this subscale.

Jason et al. (2010c) found that Role-Emotional had the lowest threshold for both identifying individuals with CFS and identifying others who did not have this illness. However, Vitality, Social Functioning and Role-Physical have the highest threshold. In a literature review, the Vitality, Social Functioning and RolePhysical subscales best discriminated those with CFS from controls (Jason et al., 2010c). The Vitality scale measures items that assess feeling full of pep and energy, as well as those that focus on feeling worn out or tired. Social functioning is assessed by items that involve interference with your normal social activities with family, friends, neighbors or groups. The RolePhysical subscale focuses on items assessing the need to cut down or limit one's work, social, or other activities, as well as accomplishing less than one might like. In other words, these three subscales capture significant limitations in the person's ability to have energy and accomplish activities in life. To meet the substantial reduction criteria, we now stipulate that at a minimum, a person needs to meet criteria on two of the three designated subscales: Score less than or equal to 35 for Vitality, less than or equal to 62.5 for Social Functioning, or less than or equal to 50 for RolePhysical. The substantial reduction criteria must be met in order for the fatigue criterion of the Revised Canadian ME/CFS case definition to be met.

Post-exertional malaise and/or post-exertional fatigue: With activity (it need not be strenuous and may include walking up a flight of stairs, using a computer, reading a book or other activities of daily living such as dressing, bathing, eating, cooking, or shopping), there must be a loss of physical or mental stamina, rapid/sudden muscle or cognitive fatigability, post-exertional malaise and/or post-exertional fatigue and a tendency for other associated symptoms within the patient's cluster of symptoms to worsen. The recovery is slow, often taking 2-24 hours or longer.

Although a person with ME/CFS may feel better after rest, he or she may get sick again quickly upon minimal activity or exertion. In this case, the rest does not completely eliminate the syndrome but it may provide some relief; therefore, this symptom pattern should not exclude a ME/CFS diagnosis. In the case of an adult who is active (e.g., participates in extracurricular activities, sports, outings with friends) but exhausted, yet also recovers quickly when activity is decreased, the fatigue would be considered the result of ongoing exertion or activity and thus, would be excluded from a diagnosis of ME/CFS. However, a person who participates in very little activity (possibly to minimize ME/CFS symptoms) when compared to his or her same-age peers and who also becomes exhausted upon minimal exertion, would meet the post-exertional malaise criterion. In summary, normal fatigue is not activity limiting, whereas the fatigue/energy problems present in ME/CFS limits the individual's activity to varying degrees. A person experiences post-exertional malaise when recovery does not occur quickly after exhausting activity or when activities have to be restricted to avoid experiencing fatigue/energy problems, or when increased fatigue and/or worsening of symptoms are experienced after exercise.

Within a group of individuals diagnosed with CFS, Jason et al. (1999a) found that post-exertional fatigue or malaise for individuals with CFS ranged from 93.8$40.6 \%$ depending on how the question was asked. There is certainly a need to standardize the questions used to reduce this source of unreliability. In addition, length of the period of post-exertional malaise may vary from activity to activity or by time period within the person. It would be more useful to subgroup patients into the following groups rather than to discount the complaints of patients who do not report experiencing this symptom for 24 hours or longer: Post-exertional malaise lasting for 1-6 hours; post-exertional malaise lasting for 7-23 hours; post-exertional malaise lasting for 24 hours or longer, with exact length specified by patient.

Recently, Jason et al. (2009b) developed the ME/CFS Fatigue Types Questionnaire (MFTQ), a 22 item scale designed to measure the duration, severity and frequency of different fatigue-related sensations and symptoms. The MFTQ appears to be a reliable and 
valid measure of fatigue types in individuals with ME/CFS. When factor analyzed, several fatigue factors emerged for individuals with CFS (Post-Exertional, Wired, Brain Fog, Energy and Flu-Like fatigue) but the healthy control group only experienced one overall factor involving generalized fatigue. Jason et al. (2010d) found that the post-exertional factor had adequate sensitivity and specificity (it was able to identify individuals who had ME/CFS and also exclude individuals who did not have this illness). Five items from the MFTQ with the highest loadings on the postexertional factor included: Dead, heavy feeling that occurs quickly after starting to exercise; next day soreness or fatigue after non-strenuous, everyday activities; mentally tired after the slightest effort; physically drained or sick after mild activity and minimum exercise makes you physically tired (Jason et al. 2009b). We included these five items on the DePaul Symptom Questionnaire to measure postexertional malaise. To meet criteria for post-exertional malaise or post-exertional fatigue, one of these items need to be endorsed at sufficient frequency and severity ( 2 or greater on a scale of $0-4$ ). This number of items was confirmed as having the best sensitivity and specificity (Jason et al., 2010d).

Sleep: The third symptom category is unrefreshing sleep, or disturbance of sleep quantity or rhythm. As discussed above, many of the symptom criteria lack clear guidelines for clinicians to follow when conducting an assessment. For example, there are no guidelines for how the term "unrefreshing sleep" should be defined and evaluated. Research findings indicate that tiredness is associated with any disturbance in sleep such as too much sleep, sleep at the wrong time and fragmented sleep. Patients with ME/CFS often report unrefreshing, disturbed, or poor quality sleep; however, the Fukuda et al. (1994) case definition does not adequately define "nonrestorative sleep" or specify how this complaint should be assessed. It is unclear whether this symptom would be counted for individuals who report nonrestorative sleep for most but not all nights. Additionally, it is not specifically stated if someone would meet this criterion if he or she has restorative sleep but other sleep problems such as difficulties falling asleep, maintaining sleep, or waking up early. Komaroff et al. (1996) found that $98 \%$ of a chronically fatigued group had sleep disturbances, but only $89 \%$ reported awakening unrefreshed. To assess sleep difficulties, it is important to assess for dysfunction in areas beyond just nonrestorative sleep and include prolonged sleep (including frequent naps), disturbed sleep (e.g., inability to fall asleep or early awakening) and/or day/night reversal. We have included items in the DePaul Symptom Questionnaire that assess several symptom indicators of sleep dysfunction and one symptom needs to meet the frequency and severity ratings of 2 or higher to meet the sleep criterion.

Myofascial pain, joint pain, abdominal and/or head pain: The fourth symptom category requires that the person exhibit myofascial pain, joint pain, abdominal and/or head pain. Myofascial pain can include deep pain, abdomen/stomach pain, or achy and sore muscles. Pain, stiffness, or tenderness may occur in any joint but must be present in more than one joint and lacking edema or other signs of inflammation. The pain criterion is met if a person meets the frequency and severity ratings of 2 or higher for one pain symptom.

Pain is among the most frequently cited reasons for seeking medical attention. However, the assessment of pain is particularly difficult given that the experience of this complaint is highly subjective. For example, pain is difficult to describe and different descriptions may be used by two different individuals to describe the same phenomenon (Turk and Melzack, 1992). Furthermore, the association between physical abnormalities and patients report of pain is often ambiguous and weakly correlated. Physical pathology also has not been found to be predictive of disability (Cats-Baril and Frymoyer, 1991). In fact, there are no isomorphic relationships between reports of pain, disability and tissue pathology. Given the inherent subjectivity of pain and the frequent absence of objective findings to account for this symptom, pain can only be assessed indirectly (Turk and Melzack, 1992). It is recommended that complaints of joint pain and muscle pain be carefully distinguished to ensure accurate reporting of symptoms. Complaints of muscle pain should be described as sensations of pain or aching experienced in the muscles and they should be distinguished from feelings of weakness or pain experienced in other areas such as the joints (Sharpe et al., 1991).

Headaches can occur in a variety of different forms and are typically classified into the following categories: vascular headaches (e.g., migraine, cluster, hypertensive), muscle contraction headaches (e.g., chronic myositis, cervical osteoarthritis, depressive equivalents and conversion reactions) and traction and inflammatory headaches (e.g., mass lesions, temporomandibular joint, occlusive vascular disease). It is not sufficient to simply ask whether the patient has had a previous or lifelong problem with headaches. Such a question is likely to result in inaccurate information because it is not specific enough. Assessment includes whether the headaches the patient 
is reporting are (1) more frequent than they were before, which would indicate new pattern, (2) of a new type as compared to headaches previously experienced (i.e., location of pain has changed, nature of pain has changed) and (3) different in severity type as compared to headaches previously experienced by the patient. If a patient endorses these three criteria and indicates their headaches meet the frequency and severity cutoffs, then this report of headaches would be counted toward fulfilling the pain criterion.

Neurological/cognitive manifestations: The fifth symptom category is the occurrence of two or more neurological/cognitive manifestations at frequency and severity ratings of 2 or higher. People with CFS typically complain that fatigue affects their physical and mental functioning. Generally, patients report problems with concentration and absent mindedness, impaired memory, difficulty making decisions, difficulties taking in written or spoken material and executing familiar sequences of events, such as grocery shopping (Wearden and Appleby, 1997). In addition, many patients with CFS complain that performing mental work is aversive for them, to the degree that they either stop doing it or start to experience symptoms (Wood et al., 1994). Aside from cognitive manifestations, many people experience neurological symptoms such as perceptual disturbances (e.g., inability to focus vision and attention), motor disturbances (e.g., muscle weakness and twitches) and overload phenomenon (e.g., sensitivity to light or sound) (Carruthers et al., 2003). As a result, many people with CFS describe their neurological/cognitive difficulties as one of the more disabling and troubling symptoms of their illness.

Autonomic, neuroendocrine, or immune manifestations: Finally, the sixth symptom category requires at least one symptom at frequency and severity ratings of 2 or higher from two of the following three subcategories: (1) Autonomic manifestations, (2) Neuroendocrine manifestations and (3) Immune manifestations.

Autonomic manifestations: Autonomic manifestations include neurally mediated hypotension, postural orthostatic tachycardia, delayed postural hypotension, palpitations with or without cardiac arrhythmias, dizziness, feeling unsteady on the feet, disturbed balance, shortness of breath, nausea, bladder dysfunction and irritable bowel syndrome.

Neuroendocrine manifestations: Neuroendocrine manifestations include recurrent feelings of feverishness and cold extremities, subnormal body temperature and marked diurnal fluctuations, sweating episodes, intolerance of extremes of heat and cold, marked weight change-loss of appetite or abnormal appetite and worsening of symptoms with stress.

Immune manifestations: Immune manifestations include recurrent flu-like symptoms, non-exudative sore or scratchy throat, repeated fevers and sweats, lymph nodes tender to palpitation--generally minimal swelling noted and new sensitivities to food, odors, or chemicals. Sore throat is one immune manifestation that is often difficult to assess because of its subjective nature. While physical findings are sometimes present and can provide objective evidence of this symptom, often times such findings are not present. According to a study conducted by Gerber et al. (1984), sore throat is the third most common reason for patient visits to primary care practitioners. Sore throat is most commonly associated with pharyngitis, the inflammation of the pharynx and surrounding lymphoid tissue. There are numerous other conditions, however, that may also produce sore throat. These conditions range from local disorders (e.g., ear, throat, nose infections) to systemic disorders (e.g., CFS, rheumatoid arthritis, viral hepatitis) and include infectious and non-infectious etiologies (Muhrer, 1991).

Medical and psychiatric evaluation: It is critical to do a medical evaluation in order to identify exclusionary medical diagnoses that would explain the fatigue and symptoms (Fukuda et al., 1994). Medical diagnoses that have been adequately treated (e.g., Lyme disease) or that are not likely to cause fatigue should not be considered exclusionary. Table 1 lists exclusionary medical conditions as well as those that should not necessarily be considered exclusionary and may present comorbidly with ME/CFS.

Psychiatric evaluation is essential to rule out psychiatric diagnoses that may be the cause of fatigue and preclude a diagnosis of CFS. The Structured Clinical Interview for the DSM-IV (SCID) (Spitzer et al., 1995), a semi-structured psychiatric interview, should be used for psychiatric evaluation. Test-retest reliability was assessed for the SCID yielding good Kappa scores (Williams et al., 1992). The professionally administered SCID allows for clinical judgment in the assignment of symptoms to psychiatric or medical categories, a crucial distinction in the assessment of symptoms that overlap between CFS and psychiatric disorders, e.g., fatigue, concentration difficulty and sleep disturbance. A psychodiagnostic study (Taylor and Jason, 1998) validated the use of the SCID in a sample of CFS patients. 
Exclusionary psychiatric conditions are noted in Table 1. Major Depressive Disorder with melancholic or psychotic features are considered exclusionary conditions in our criteria, primarily due to the findings that melancholic and psychotic processes represent distinct biological or endocrinological processes and may respond well to antidepressant or antipsychotic medications (Robbins et al., 1989; Schulkin, 1994). Reeves et al. (2003) has recommended that Major Depressive Disorder with melancholic features, anorexia nervosa, or bulimia, not be considered exclusionary if these conditions have been resolved for more than 5 years before the onset of the current illness. However, for our ME/CFS case definition, eating disorders (i.e., anorexia nervosa and bulimia nervosa) and substance abuse are exclusionary only if the diagnosis is current. A diagnosis of melancholic depression, substance abuse or eating disorder that has been appropriately treated and resolved should not be considered exclusionary. We believe that psychotic disorders of any variety continue to be exclusionary.

Predating criteria: The provision that the symptoms such as sore throat or memory impairment not predate the fatigue has also been modified. Some individuals who develop ME/CFS experience a prodromal phase, in which symptoms begin to appear in the year prior to the onset of fatigue. Jason et al. (1999a) compared two groups of patients, those with CFS and those with chronic fatigue due to psychiatric reasons. Using the two Fukuda et al. (1994) criteria of having six or more months of fatigue and the symptom not predating the illness, only one of eight Fukuda symptoms were significantly different between the two groups. However, if one did not count whether a symptom predated the illness and used both the 6 month criteria as well as only counting moderate to serious manifestations of the illness (as recommended in our current case definition), then four out of the eight symptoms were significantly differentiated between the two groups. There were significantly higher symptoms for the CFS group versus the psychiatric fatigued group for muscle pain $(46.9 \%$ versus $24.2 \%)$, headaches (50.0\% versus $21.2 \%$ ), impaired memory/concentration $(65.6 \%$ versus $30.3 \%)$ and unrefreshing sleep $(78.1 \%$ versus $39.4 \%$ ). Therefore, it is not whether a symptom occurred before the illness, but the issue of severity of symptoms that is the best discriminator of those with CFS versus those who have a psychiatric reason for their fatigue. In our revised case definition, we count all symptoms even if they occurred prior to the onset of the fatigue.
Meeting research versus clinical criteria: Table 1 provides all the symptoms as specified in the Revised Canadian ME/CFS case definition. Some meet full criteria whereas others who are very symptomatic do not meet full criteria. We argue as we did with the Pediatric case definition (Jason et al., 2006) that those that meet full criteria are more homogenous and might be best used for research purposes and we now classify these individuals as meeting the Research ME/CFS criteria. Still, others might have the illness but not meet one of the required criteria. We classified such individual as meeting Clinical ME/CFS criteria. These individuals needed to have six or more months of fatigue and needed to report symptoms in five out of the six ME/CFS symptom categories (one of which has to be post exertional malaise, as it is critical to this case definition). In addition, for autonomic, neuroendocrine and immune manifestations, adults must have at least one symptom in any of these three categories, as opposed to one symptom from two of the three categories. We also have a category called Atypical ME/CFS, which is defined as six or more months of fatigue, but having two to four ME/CFS symptoms. There is also a category called ME/CFS-Like, which involves exhibiting all criteria categories but for a duration of fewer than 6 months. Further, a person could be classified as having $\mathrm{ME} / \mathrm{CFS}$ in remission if the person had previously been diagnosed with CFS by a physician but was not currently meeting the Research ME/CFS Criteria, Clinical ME/CFS criteria, or Atypical ME/CFS criteria and must have 0 or 1 classic ME/CFS symptoms.

\section{CONCLUSION}

In this study, we present the Revised Canadian ME/CFS case definition, which provides greater specification to the work of Carruthers et al. (2003). The scientific enterprise depends on reliable and valid ways of classifying patients into diagnostic categories and this critical research activity can enable investigators to better understand etiology, pathophysiology and treatment approaches for CFS and other disorders (King and Jason, 2005). When diagnostic categories lack reliability and accuracy, the quality of treatment and clinical research can be significantly compromised. A misdiagnosis may lead to improper treatment and in cases of severe illness, the matter of an incorrect diagnosis can have serious consequences. In other words, the validity (i.e., usefulness) of a diagnostic category is inherently limited by its reliability. Therefore, to the extent to which a diagnostic category is unreliable, a limit is placed on its validity for any clinical research 
(Spitzer et al., 1975). The poor understanding of the pathophysiology of ME/CFS may be due case definitions lacking reliabiltiy and validity and improving the case definition may prevent complications in identifying biological markers in this illness. Issues concerning reliability of clinical diagnosis are therefore complex and have important research and practical implications (Jason et al., 2005).

One of the greatest sources of diagnostic unreliability is criterion variance, which is differences in the formal inclusion and exclusion criteria used by clinicians to classify patients into diagnostic categories (Spitzer et al., 1975). Cantwell (1996) purports that diagnostic criteria should specify which diagnostic instrument to use, what informants to use and how to rate for presence and severity of the criteria. For example, one might specify that a certain number and type of symptoms should be present in order to make a particular diagnosis. In addition to the importance of the number and type of symptoms, definitions of fatigue should also include specific guidelines pertaining to the importance of symptom severity in the diagnostic procedure. Given the high variability in symptom severity among persons with fatigue, standardized procedures need to be employed for determining whether or not a particular symptom is severe enough to qualify for the diagnosis of fatigue.

Although brief periods of fatigue (i.e., less than 1 month duration) occur in approximately $15-25 \%$ of the population, chronic fatigue occurs in about $4-5 \%$ of the population (Jason et al., 2010b). In a community-based epidemiologic study of fatigue (Jason et al., 1999b), of those identified with six or more months of fatigue, $54 \%$ had chronic fatigue explained by medical or psychiatric reasons, including cancer, multiple sclerosis and melancholic depression. If about $5 \%$ of the population has six or more months of fatigue and about half of this is due to clear medical or psychiatric reasons, then the critical question is how many of the remaining $2.5 \%$ have ME/CFS? The empiric CFS case definition (Reeves et al., 2005) estimates that $2.54 \%$ do have this illness, so that research group would suggest that almost all would fall within the CFS category. However, Jason et al. (2009b) believe that within this $2.54 \%$ are mood disorders, which are one of the most prevalent psychiatric disorders (1 month prevalence rate of major depressive episode is 2.2\%) (Regier et al., 1988). As an example, Major Depressive Disorder (MDD) can be confused with ME/CFS because it has some overlapping symptoms with the illness. It is possible that some patients with MDD also have chronic fatigue and four Fukuda et al. (1994) symptoms that can occur with depression (e.g., unrefreshing sleep, joint pain, muscle pain, impairment in concentration). Consequently, it is possible that some patients with a primary mood disorder could be misdiagnosed as having ME/CFS. Yet, these are distinct illnesses, as several ME/CFS symptoms are not commonly found in depression, including prolonged fatigue after physical exertion, night sweats, sore throats and swollen lymph nodes. Illness onset with ME/CFS often occurs over a few hours or days, whereas primary depression generally shows a more gradual onset. Biological findings also differentiate the two conditions (Jason et al., 2005). The inclusion of the latter type of patients in the current ME/CFS case definition could confound the interpretation of epidemiologic and treatment studies; therefore, complicating efforts to identify biological markers for this illness.

The DePaul Symptom Questionnaire is a useful screening tool to assess for ME/CFS according to the Revised Canadian ME/CFS case definition, but it does not provide the full picture of a patient's symptomatology. Thus, for research purposes, we propose some additional measures that could be administered to obtain more comprehensive data on symptomatology. For fatigue, the Fatigue Severity Scale (FSS) (Krupp et al., 1989) is a measure of the behavioral consequences of fatigue. In a study by Jason et al. (2010e), the FSS was found to have a better ability to detect cases and non-cases than the MFI (Smets et al., 1995), the Fatigue Scale (Chalder et al., 1993) and the Profile of Fatigue-Related Symptoms (Ray et al., 1992). For sleep disturbances, we suggest the Pittsburgh Sleep Quality Index (Buysse, 1989) for measuring sleep disruptions and sleep quality. Finally, pain symptoms can be assessed with the McGill Pain Questionnaire, a well-validated measure (Melzack, 1975).

Fukuda et al. (1994) recommended subgrouping adult CFS patients and while not the focus of this article, similar efforts suggest this would be appropriate in the study of ME/CFS (Jason et al., 2005). Cancer and heart disease are comprised of many subtypes and this may also be the case with ME/CFS. Although ME/CFS has also been referred to as unexplained fatigue, this is also changing with scientific discoveries. For research purposes, each of the six domains that are assessed with the Revised Canadian criteria may be operationalized in more objective and precise ways in the future. For the fatigue criterion, efforts are ongoing to measure actual behavioral abnormalities among ME/CFS patients using actigraphs (Tryon et al., 2004). Post-exertional fatigue and pain can be measured by increases in the expression for sensory, adrenergic and immune genes following moderate exercise (Light et al., 2009). 
Unrefreshing sleep or disturbance of sleep quantity or rhythm disturbance will be measured by polysomnography (Shaver, 2003). Neurological/cognitive manifestations could be measured by objective evidence using functional Magnetic Resonance Imaging (fMRI) (Lange et al., 2005). Autonomic manifestations could be assessed by ejection fraction decreases, suggesting left ventricular dysfunction in the heart (Peckerman et al., 2003). Neuroendocrine manifestations can be measured by abnormal levels of circulating cortisol (Torres-Harding et al., 2008). Finally, immune manifestations could be measured by elevations in CD5+CD19+ subset and decreased natural killer cell cytotoxicity (Maher et al., 2003).

In conclusion, studies examining sources of diagnostic unreliability have shown that criterion variance, differences in the formal inclusion and exclusion criteria used by clinicians to classify patients' data into diagnostic categories, accounts for the largest source of diagnostic unreliability (Spitzer et al., 1975). Moreover, research has demonstrated that criterion variance is most likely to occur when operationally explicit criteria do not exist for diagnostic categories (Spitzer et al., 1978). The provision of either a structured or semi-structured standardized instrument designed to elicit the required information (formal inclusion and exclusion criteria) would greatly reduce this source of variance and improve the diagnostic reliability of $\mathrm{CFS}$. If $\mathrm{ME} / \mathrm{CFS}$ is to be diagnosed reliably across health care professionals, we believe that it is imperative to provide specific thresholds and scoring rules for the symptom criteria. Without such standardization, symptom variability will be a function of the assessment procedure and etiological factors. In other words, by determining specific thresholds and scoring rules for the symptom criteria, variability is likely to result in increased diagnostic reliability.

\section{REFERENCES}

Acheson, E.D., 1959. The clinical syndrome variously called benign Myalgic Encephalomyelitis, Iceland disease and epidemic neuromyasthenia. Am. J. Med., 26: 569-95. PMID: 13637100

Buysse, D.J., 1989. The Pittsburgh sleep quality index: A new instrument for psychiatric practice and research. Psychiatr. Res., 28: 193-213. PMID: 2748771

Cantwell, D.P., 1996. Classification of child and adolescent psychopathology. J. Child Psychol. Psychiatry, 37: 3-12. PMID: 8655656.
Carruthers, B.M., A.K. Jain, K.L. DeMeirleir, D.L. Peterson and N.G. Klimas et al., 2003. Myalgic Encephalomyelitis/chronic fatigue syndrome: Clinical working case definition, diagnostic and treatments protocols. J. Chronic Fatigue Syndr., 11: 7-115. DOI: 10.1300/J092v11n01_01

Cats-Baril, W. and J. Frymoyer, 1991. Identifying patients at risk of becoming disabled because of low back pain. Spine, 16: 605-607. PMID: 1830688

Chalder, T., G. Berelowitz, T. Pawlikowska, L. Watts and S. Wessley et al., 1993. Development of a fatigue scale. J. Psychosom. Med., 37: 147-153.

Corradi, K.M., L.A. Jason and S.R. Torres-Harding, 2006. Exploratory Subgrouping in CFS: Infectious, Inflammatory and Other. In: Advances in Psychology Research, Columbus, A. (Eds.). Hauppauge, Nova Science Publishers, New York, ISBN: 1-60021-105-4, pp: 115-127.

Dowsett, E.G., A.M. Ramsay, R.A. McCartney and E.J. Bell, 1990. Myalgic encephalomyelitis-a persistent enteroviral infection? Postgrad. Med. J., 66: 526-530. PMID: 2170962

Dowsett, E.G., E.M. Goudsmit, A. Macintyre and C. Shepherd, 1994. London Criteria for Myalgic Encephalomyelitis. In: Report from the National Task Force on Chronic Fatigue Syndrome (CFS), Post Viral Fatigue Syndrome (PVFS), Myalgic Encephalomyelitis (ME), Tyrrell, D. (Ed.). Westcare, Clifton, pp: 96-98.

Friedberg, F., L. Dechene, M. McKenzie and R. Fontanetta, 2000. Symptom patterns in long-term chronic fatigue syndrome. J. Psychosom. Res., 48: 59-68. DOI: 10.1016/S0022-3999(99)00077-X

Fukuda, K., S.E. Straus, I. Hickie, M.C. Sharpe and J.G. Dobbins et al., 1994. The chronic fatigue syndrome: A comprehensive approach to its definition and study. Ann. Intern. Med., 121: 953-959. PMID: 7978722

Gerber, M., R.W. Ryan, R.C. Tilton and J.E. Watson, 1984. Role of Chlamydia trachomatis in acute pharyngitis in young adults. J. Clin. Microbiol., 20: 993-994. PMID: 6511881

Goudsmit, E., C. Shepherd, C.P. Dancey and S. Howes, 2009. ME: Chronic fatigue syndrome or a distinct clinical entity? Health Psychol. Update, 18: 26-31. PMID: 19576714

Holmes, G.P., J.E. Kaplan, N.M. Gantz, A.L. Komaroff and L.B. Schonberger et al., 1988. Chronic fatigue syndrome: A working case definition. Ann. Intern. Med., 108: 387-389. PMID: 2829679 
Hyde, B., 1999. Are Myalgic encephalomyelitis and chronic fatigue syndrome synonymous? Syndr. Sentinel., 3: 5-8.

Hyde, B.M., J.A. Goldstein and P. Levine, 1992. The Clinical and Scientific Basis of Myalgic Encephalomyelitis/Chronic Fatigue Syndrome. Nightingale Research Foundation, Canada, ISBN: 10: 0969566204 , pp: 724.

Janal, M.N., D.S. Ciccone and B.H. Natelson, 2006. Sub-typing CFS patients on the basis of 'minor' symptoms. Biol. Psychol., 73: 124-31. PMID: 16473456

Jason, L.A., N. Porter, E. Shelleby, L. Till and D.S. Bell et al., 2010a. Examining criteria to diagnose ME/CFS in pediatric samples. (In Press).

Jason, L.A., M. Evans, M. Brown and N. Porter, 2010b. What is fatigue? Pathological and non-pathological fatigue. Acad. Phys. Med. Rehabil, 2: 327-331. DOI: 10.1016/j.pmrj.2010.03.028

Jason, L.A., M. Brown, M. Evans, V. Anderson, A. Lerch, A. Brown et al., 2010c. Measuring substantial reduction in functioning with CFS. (In Press).

Jason, L.A., M. Evans, M. Brown, N. Porter and A. Brown et al., 2010d. Fatigue scales and chronic fatigue syndrome: Issues of sensitivity and specificity. (In Press).

Jason, L.A., M. Evans, A. Brown, M. Brown and N. Porter et al., 2010e. Sensitivity and specificity of the CDC empirical chronic fatigue syndrome case definition. Psychology, 1: 9-16. DOI: 10.4236/psych.2010.11002

Jason, L.A., C.P. King, J.A. Richman, R.R. Taylor and S.R. Torres et al., 1999a. US case definition of chronic fatigue syndrome: Diagnostic and theoretical issues. J. Chronic Fatigue Syndr., 5: 3-33. DOI: 10.1300/J092v05n03_02

Jason, L.A., J.A. Richman, A.W. Rademaker, K.M. Jordan and A.V. Plioplys et al., 1999b. A communitybased study of chronic fatigue syndrome. Arch. Intern. Med., 159: 2129-2137. PMID: 10527290

Jason, L.A. and M. Choi, 2008. Dimensions and Assessment of Fatigue. In: Fatigue Science for Human Health, Watanabe, Y., B. Evengard, B.H. Natelson, L.A. Jason and H. Kuratsune (Eds.). Springer, Tokyo, ISBN: 978-4-431-73463-5, pp: 1-16.

Jason, L.A. and R.R. Taylor, 2002. Applying cluster analysis to define a typology of chronic fatigue syndrome in a medically-evaluated, random community sample. Psychol. Health, 17: 323-337. DOI: $10.1080 / 08870440290029575$
Jason, L.A., J. Helgerson, S.R. Torres-Harding, A.W. Carrico and R.R. Taylor, 2003. Variability in diagnostic criteria for chronic fatigue syndrome may result in substantial differences in patterns of symptoms and disability. Eval. Health Prof, 26: 3-22. PMID: 12629919

Jason, L.A., K. Corradi and S. Torres-Harding, 2007. Toward an empirical case definition of CFS. J. Soc. Serv. Res., 34: 43-54. DOI: 10.1300/J079v34n02_04

Jason, L.A., K. Corradi, S. Torres-Harding, R.R. Taylor and C. King, 2005. Chronic fatigue syndrome: The need for subtypes. Neuropsychol. Rev., 15: 29-58. DOI: $10.1007 / \mathrm{s} 11065-005-3588-2$

Jason, L.A., N. Najar, N. Porter and C. Reh, 2009a. Evaluating the centers for disease control's empirical chronic fatigue syndrome case definition. J. Disabil. Policy Stud., 20: 93-100. DOI: 10.1177/1044207308325995

Jason, L.A., T. Jessen, N. Porter, A. Boulton and M.G. Njoku et al., 2009b. Examining types of fatigue among individuals with ME/CFS. Disability Stud. Q., 29. http://www.dsqsds.org/article/view/938/1113

Jason, L.A., R.R. Taylor, C.L. Kennedy, K. Jordan and C. Huang et al., 2002a. A factor analysis of chronic fatigue symptoms in a community-based sample. Soc. Psychiat. Psychiatr. Epidemiol., 37: 183-189. DOI: $10.1007 / \mathrm{s} 001270200013$

Jason, L.A., S.R. Torres-Harding, A.W. Carrico and R.R. Taylor, 2002b. Symptom occurrence with chronic fatigue syndrome. Biol. Psychol., 59: 15-27. DOI: 10.1016/S0301-0511(01)00120-X

Jason, L.A., S.R. Torres-Harding, R.R. Taylor and A.W. Carrico, 2001. A comparison of the 1988 and 1994 diagnostic criteria for chronic fatigue syndrome. J. Clin. Psychol. Med. Sett., 8: 337-343. DOI: $10.1023 / A: 1011981132735$

Jason, L.A., S.R. Torres-Harding, A. Jurgens and J. Helgerson, 2004. Comparing the Fukuda et al. criteria and the Canadian case definition for chronic fatigue syndrome. J. Chronic Fatigue Syndr., 12: 37-52. DOI: 10.1300/J092v12n01_03

Jason, L.A., D.S. Bell, K. Rowe, E.L.S. Van Hoof and K. Jordan et al., 2006. A pediatric case definition for ME/CFS. J. Chronic Fatigue Syndr., 13: 1-44.

Katon, W. and J. Russo, 1992. Chronic fatigue syndrome criteria. A critique of the requirement for multiple physical complaints. Arch. Intern. Med., 152: 1604-1609. DOI: 10.1300/J092v13n02_01

Katon, W.J., D.S. Buchwald, G.E. Simon, J.E. Russo and P.J. Mease, 1991. Psychiatric illness in patients with chronic fatigue syndrome and rheumatoid arthritis. J. Gen. Intern. Med., 6: 277-285. DOI: 10.1007/BF02597420 
King, C.P. and L.A. Jason, 2005. Improving the diagnostic criteria and procedures for chronic fatigue syndrome. Biol. Psychol., 68: 87-106. DOI: 10.1016/J.BIOPSYCHO.2004.03.015

Komaroff, A.L., L.R. Fagioli, A.M. Geiger, T.H. Doolittle and J. Lee et al., 1996. An examination of the working case definition of Chronic Fatigue Syndrome. Am. J. Med., 100: 56-64. DOI: 10.1016/S0002-9343(96)90012-1

Krupp, L.B., N.G. LaRocca, J. Muir-Nash and A.D. Steinberg, 1989. The fatigue severity scale: Application to patients with multiple sclerosis and systemic lupus erythematosus. Arch. Neurol., 46: 1121-1123. PMID: 2803071

Lange, G., J. Steffner, D.B. Cook, B.M. Bly and C. Christodoulou et al., 2005. Objective evidence of cognitive complaints in chronic fatigue syndrome: A BOLD fMRI study of verbal working memory. Neuroimage, 26: 513-524. PMID: 15907308

Light, A.R., A.T. White, R.W. Hughen and K.C. Light, 2009. Moderate exercise increases expression for sensory, adrenergic and immune genes in chronic fatigue syndrome patients but not in normal subjects. J. Pain, 10: 1099-1112. PMID: 19647494

Lloyd, A.R., I. Hickie, C.R. Boughton, O. Spencer and D. Wakefield, 1990. Prevalence of chronic fatigue syndrome in an Australian population. Med. J. Aust., 153: 522-528. PMID: 2233474

Maher, K.J., N.G. Klimas and M.A. Fletcher, 2003. Immunology. In: Handbook of Chronic Fatigue Syndrome, Jason, L.A., P.A. Fennell and R.R. Taylor (Eds.). Wiley, New York, ISBN: 0-471-41512-X, pp: 124-151.

Melzack, R., 1975. The McGill pain questionnaire: Major properties and scoring methods. Pain, 1: 277-299. DOI: 10.1016/0304-3959(75)90044-5

Muhrer, J., 1991. Diagnostic considerations in the evaluation and treatment of sore throat. Nurse Prac., 16: 33-45. PMID: 1945075

Nisenbaum, R., M. Reyes, A.C. Mawle and W.C. Reeves, 1998. Factor analysis of unexplained severe fatigue and interrelated symptoms: Overlap with criteria for chronic fatigue syndrome. Am. J. Epidemiol., 148: 72-77. PMID: 9663406

Nisenbaum, R., M. Reyes, E.R. Unger and W.C. Reeves, 2004. Factor analysis of symptoms among subjects with unexplained chronic fatigue. What can we learn about chronic fatigue syndrome? J. Psychosom. Res., 56: 171-178. PMID: 15016574

Peckerman, A., R. Chemitiganti, C. Zhao, K. Dahl and B.H. Natelson et al., 2003. Left ventricular function in Chronic Fatigue Syndrome (CFS): Data from nuclear ventriculography studies of responses to exercise and portural stress. FASEB, 17: 853-853.
Ramsay, A.M., 1981. Myalgic Encephalomyelitis: A baffling syndrome with a tragic aftermath. The ME Association. http://www.25megroup.org/Information/Medical/b affling\%20syndrome.htm

Ramsay, A.M., 1988. Myalgic Encephalomyelitis and Postviral Fatigue States: The Saga of Royal Free Disease. 2nd Edn., Gower Publishing Co, London, pp: 68.

Ray, C., W.R.C. Weir, S. Cullen and S. Phillips, 1992. Development of a measure of symptoms in chronic fatigue syndrome: the Profile of Fatigue-Related Symptoms (PFRS). Psychol. Health, 7: 27-43. DOI: 10.1080/08870449208404293

Reeves, W.C., A. Lloyd, S.D. Vernon, N. Klimas and L. Jason et al., 2003. Identification of ambiguities in the 1994 chronic fatigue syndrome research case definition and recommendations for-resolution. BMC Health Serv. Res., 3: 25-25. DOI: 10.1186/1472-6963-3-25

Reeves, W.C., D. Wagner, R. Nisenbaum, J.F. Jones and B. Gurbaxani et al., 2005. Chronic fatigue syndrome-a clinical empirical approach to its definition and study. BMC. Med., 3: 19-19. DOI: 10.1186/1741-7015-3-19

Reeves, W.C., J.J. Jones, E. Maloney, C. Heim and D.C. Hoaglin et al., 2007. New study on the prevalence of CFS in metro, urban and rural Georgia populations. Popul. Health Metrics, 5: 55. DOI: 10.1186/1478-7954-5-5

Regier, D.A, J.H. Boyd and J.D. Burke Jr., 1988. Onemonth prevalence of mental disorders in the United States: Based on five epidemiological catchment area sites. Arch. Gen. Psychiatry, 45: 977-986. PMID: 3263101

Reyes, M, R. Nisenbaum D.C. Hoaglin E.R. Unger and C. Emmons et al., 2003. Prevalence and incidence of chronic fatigue syndrome in Wichita, Kansas. Arch. Intern. Med., 163: 1530-1536. PMID: 12860574

Robbins, D.R., N.E. Alessi and M.V. Colfer, 1989. Treatment of adolescents with major depression: Implications of the DST and the melancholic clinical subtype. J. Affect. Disord., 17: 99-104. PMID: 2527896

Rowe, K.S. and K.J. Rowe, 2002. Symptom Patterns of Children and Adolescents with Chronic Fatigue Syndrome. In: International Perspectives on Child and Adolescent Mental Health, Singh, N.N., T.H. Ollendick and A.N. Singh (Eds.). Elsevier Science Ltd., Oxford, ISBN: 0-08-043861-X, pp: 395-421.

Schluederberg, A., S.E. Straus, P. Peterson, S. Blumenthal and A.L. Komaroff et al., 1992. Chronic fatigue syndrome research: Definition and medical outcome assessment. Ann. Intern. Med., 117: 325-331. PMID: 1322076 
Schulkin, J., 1994. Melancholic depression and the hormones of adversity: A role for the amygdala. Curr. Dir. Psychol. Sci., 3: 41-44. DOI: 10.1111/j.1467-8721.1994.tb00140

Sharpe, M.C., L.C. Archard, J.E. Banatvala, L.K. Borysiewicz and A.W. Clare et al., 1991. A report-Chronic fatigue syndrome: Guidelines for research. J. R. Soc. Med., 84: 118-121. PMID: 1999813

Shaver, J.L., 2003. Sleep Disorders. In: Handbook of Chronic Fatigue Syndrome, Jason, L.A., P.A. Fennell and R.R. Taylor (Eds.). Wiley, New York, ISBN: 0-471-41512-X, pp: 281-303.

Smets, E.M., B.J. Garssen, B. Bonke and J.C. DeHaes, 1995. The Multidimensional Fatigue Inventory (MFI) psychometric properties of an instrument to assess fatigue. J. Psychosom. Res., 39: 315-325. PMID: 7636775

Spitzer, R., J. Endicott and E. Robins, 1975. Clinical criteria for psychiatric diagnosis and DSM-III. Am. J. Psychiatry, 132: 1187-1192. PMID: 1172654

Spitzer, R., J. Endicott and E. Robins, 1978. Research diagnostic criteria. Arch. Gen. Psychiatry, 35: 773-782.

Spitzer, R.L., J.B.W. Williams, M. Gibbon and M.B. First, 1995. Structured Clinical Interview for DSM-IV TM Source Book. 2nd Edn., American Psychiatric Press, Washington DC., ISBN: 089042-071-8, pp: 1232.

Stouten, B., 2005. Identification of ambiguities in the 1994 chronic fatigue syndrome research case definition and recommendations for resolution. BMC. Health Serv. Res., 5: 37-37. DOI: 10.1186/1472-6963-5-37.

Sullivan, P.F., N.L. Pedersen, A. Jacks and B. Evengard, 2005. Chronic fatigue in a population sample: Definitions and heterogeneity. Psychol. Med., 35: 1337-1348. PMID: 16168156

Taylor, R.R. and L.A. Jason, 1998. Comparing the DIS with the SCID: Chronic fatigue syndrome and psychiatric comorbidity. Psychol. Health: Int. Rev. Health Psychol., 13: 1087-1104. DOI: 10.1080/08870449808407452

Tiersky, L.A., R.J. Matheis, J. DeLuca, G. Lange and B.H. Natelson, 2003. Functional status, neuropsychological functioning and mood in chronic fatigue syndrome. J. Nerv. Ment. Dis., 191: 324-331. PMID: 12819552
Torres-Harding, S., M. Sorenson, L.A. Jason, K. Maher and M.A. Fletcher et al., 2008. The associations between basal salivary cortisol levels and illness symptomatology in chronic fatigue syndrome. J. Applied Biobehav. Res., 13: 157-180. PMID: 19701493

Tryon, W.W., L.A. Jason, E. Frankenberry and S. Torres-Harding, 2004. Chronic fatigue syndrome impairs circadian rhythm of activity level. Physiol. Behav., 82: 849-853. PMID: 15451649

Turk, D. and R. Melzack, 1992. The Measurement of Pain and the Assessment of People Experiencing Pain. In: Handbook of Pain Assessment, Turk, D. and R. Melzack (Eds.). The Guildford Press, New York, ISBN: 978-1572304888, pp: 791-798.

Wagner, D., R. Nisenbaum, C. Heim, J.F. Jones and E.R. Unger et al., 2005. Psychometric properties of the CDC symptom inventory for assessment of chronic fatigue syndrome. Popul. Health Metrics, 3: 8-8. PMID: 16042777

Ware, J.E. and C.D. Sherbourne, 1992. The MOS 36item Short-Form health survey (SF-36): Conceptual framework and item selection. Med. Care, 30: 473-483. PMID: 1593914

Ware, Jr., J.E., M. Kosinski and B. Gandek, 2000. SF36 Health Survey: Manual and Interpretation Guide. 2nd Edn., Quality Metric Inc., Lincoln, RI., ISBN: 1891810065.

Wearden, A. and L. Appleby, 1997. Cognitive performance and complaints of cognitive impairment in chronic fatigue syndrome. Psychol. Med., 27: 81-90. PMID: 9122311

Williams, J.B., M. Gibbon, M.B. First, R.L. Spitzer and M. Davies et al., 1992. The Structured Clinical Interview for DSSM-III-R (SCID). II. Multisite test-retest reliability. Arch. Gen. Psychiatry, 49: 630-636. PMID: 1637253

Wood, G., R. Bentall, M. Gopfert, M. Dewey and R. Edwards, 1994. The differential response of chronic fatigue neurotic and muscular dystrophy patients to experimental psychological stress. Psychol. Med., 24: 357-364. PMID: 8084931 